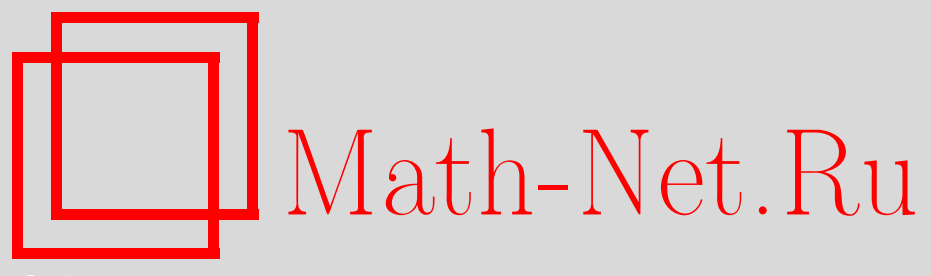

Е. В. ЖКужома, В. С. Медведев, О слоениях, определяемых гармоническими функциями, Матем. заметки, 2008, том 84, выпуск 1, 132-135

DOI: https://doi.org/10.4213/mzm4425

Использование Общероссийского математического портала Math-Net.Ru подразумевает, что вы прочитали и согласны с пользовательским соглашением http://www . mathnet.ru/rus/agreement

Параметры загрузки:

IP : 3.80 .253 .173

26 апреля 2023 г., 16:06:49

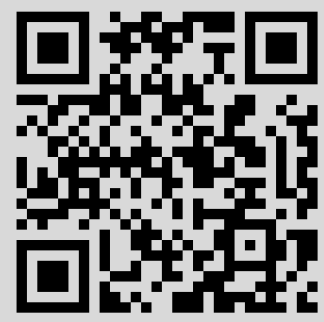




\section{О слоениях, определяемых гармоническими функциями}

\section{Е. В. Жужома, В. С. Медведев}

1. Введение. Основная цель данной заметки - привлечь внимание к одной проблеме, сформулированной Капланом в работе [1] и дать ее частичное решение. Напомним некоторые определения и зафиксируем обозначения.

Гармонические функции. Комплексная плоскость, которую иногда мы будем отождествлять с евклидовой плоскостью $\mathbb{R}^{2}$, обозначается через $\mathbb{C}$. Пусть $D \subseteq \mathbb{C}$ - некоторая область. Функция $f: D \rightarrow \mathbb{C}$ называется голоморфной, если $\partial f / \partial z$ существует для всех $z \in D$. Эквивалентное условие:

$$
\frac{\partial f}{\partial \bar{z}} \equiv 0, \quad \text { где } \quad \frac{\partial}{\partial \bar{z}}=\frac{1}{2}\left(\frac{\partial}{\partial x}+i \frac{\partial}{\partial y}\right) .
$$

Функция $f: D \rightarrow \mathbb{R}$ называется гармонической, если

$$
\triangle f \equiv 0, \quad \text { где } \quad \triangle=\frac{\partial^{2}}{\partial x^{2}}+\frac{\partial^{2}}{\partial y^{2}}=4 \frac{\partial^{2}}{\partial z \partial \bar{z}}
$$

- оператор Лапласа. Известно, что действительная и мнимая части голоморфной функции являются гармоническими функциями.

Римановы поверхности. Двумерное связное многообразие $S$ называется римановой поверхностью, если $S$ наделено комплексным атласом $\left\{U_{\alpha}, z_{\alpha}\right\}$ с биголоморфными функциями $z_{\alpha} z_{\beta}^{-1}$ (там, где они определены). Максимальный атлас задает конформную структуру на $S$. Римановы поверхности $S_{1}, S_{2}$ называются конформно эквивалентными, если существует конформный гомеоморфизм $\varphi: S_{1} \rightarrow S_{2}$ с конформным обратным.

Комплексная плоскость $\mathbb{C}$ является римановой поверхностью. Ее конформная структура определяется атласом, единственной картой которого является тождественное отображение $\mathbb{C} \rightarrow \mathbb{C}$. Любая открытая связная область $D \subseteq \mathbb{C}$ естественным образом тоже становится римановой поверхностью. Знаменитая теорема об униформизации гласит, что любая некомпактная односвязная риманова поверхность конформно эквивалентна либо $\mathbb{C}$, либо верхней полуплоскости, которая конформно эквивалентна открытому единичному диску $D_{1}=\{|z|<1\}$.

Пусть $S$ - риманова поверхность с конформной структурой $\left\{U_{\alpha}, z_{\alpha}\right\}$. Функция $f: S \rightarrow \mathbb{R}$ называется гармонической на $S$, если для любой карты $U_{\alpha}$ функция $f \circ z_{\alpha}^{-1}$ гармоническая на $z_{\alpha}\left(U_{\alpha}\right)$.

Каплан [1] доказал следующий замечательный результат: пусть $\mathscr{F}$ - слоение без особенностей на плоскости $\mathbb{R}^{2}$. Тогда существует конформная структура, превращающая $\mathbb{R}^{2}$ в риманову поверхность $S$, и гармоническая функция $f$ на $S$ такие, что слои слоения $\mathscr{F}$ являются линиями уровня функции $f$. Следуя [1], будем называть слоение $\mathscr{F}$ параболическим (соответственно гиперболическим), если $S$ конформно эквивалентна $\mathbb{C}$ (соответственно $\left.D_{1}\right)$. Каплан [1] доказал, что любое слоение без особенностей на плоскости $\mathbb{R}^{2}$ гиперболическое, и существует счетное множество гиперболических слоений попарно различных топологических типов, которые не являются параболическими. В связи с этими результатами Каплан сформулировал несколько проблем, одна из которых относилась к вопросу о мощности топологических типов параболических слоений.

Мы показываем, что существует счетное семейство попарно топологически не эквивалентных параболических слоений.

Работа выполнена при поддержке Российского фонда фундаментальных исследований (гранты №№ 08-01-00547a, 05-01-00501). 
Теорема. Существует счетное семейство попарно топологически не эквивалентных параболических слоений $\mathscr{F}_{n}$ без особенностей.

2. Доказательство теоремы. Следуя [2; § 15$]$, введем понятия орбитно-устойчивых и орбитно-неустойчивых слоев слоения (в [2] аналогичные понятия определены для траекторий потока). Далее $N_{\varepsilon}(A)$ означает $\varepsilon$-окрестность множества $A$.

Пусть на компактном метрическом пространстве $R$ задано одномерное слоение $\mathscr{F}$. Под слоем слоения мы будем понимать либо одномерный слой, либо особенность слоения (которую можно рассматривать как нульмерный слой). Пусть $x_{0}-$ точка на слое $l$ слоения $\mathscr{F}$ (если $l$-особенность, то $x_{0}=l$ ). Слой $l$ называется орбитно-устойчивым в точке $x_{0}$, если для любого $\varepsilon>0$ найдется $\delta>0$ такое, что все слои, проходящие через $N_{\delta}\left(x_{0}\right)$, лежат в $N_{\varepsilon}(l)$. Из теоремы о непрерывной зависимости слоев от начальных условий вытекает, что если слой орбитно-устойчив в одной точке, то он орбитно-устойчив в любой своей точке. Поэтому можно говорить об орбитно-устойчивом слое, если он орбитно-устойчив в любой своей точке. В противном случае слой называется орбитно-неустойчивым.

Так как непрерывное отображение компактного пространства равномерно непрерывно, то имеет место следующая лемма (теорема 36 [2]; хотя формально эта теорема доказана для потоков на сфере или ограниченной области плоскости, доказательство дословно повторяется для слоений).

Лемма 1. Пусть слоения $\mathscr{F}_{1}, \mathscr{F}_{2}$ топологически эквивалентны посредством гомеоморфизма $\varphi: R \rightarrow R$. Тогда $\varphi$ переводит орбитно-устойчивые слои в орбитно-устойчивые, а орбитно-неустойчивые - в орбитно-неустойчивые.

Обозначим через $B(\mathscr{F})$ множество орбитно-неустойчивых слоев (включая, особенности) слоения $\mathscr{F}$, и предположим, что $B(\mathscr{F}) \neq \varnothing$. Ясно, что множество $B(\mathscr{F})$ инвариантное, т.е. является объединением нульмерных и (целых) одномерных слоев. Поэтому $B(\mathscr{F}) \stackrel{\text { def }}{=} R_{1}$ можно рассматривать как носитель слоения $\left.\mathscr{F}\right|_{R_{1}} \stackrel{\text { def }}{=} \mathscr{F}_{1}$. Топология на $R_{1}$ индуцируется топологией пространства $R \stackrel{\text { def }}{=} R_{0}$, как на подмножестве. Теперь можно определить множество $B\left(\mathscr{F}_{1}\right)$ орбитно-неустойчивых слоев слоения $\mathscr{F}_{1}$. Продолжая этот процесс, получим цепочку инвариантных подмножеств

$$
R_{0} \supseteq R_{1} \supseteq \cdots \supseteq R_{n} \supseteq \cdots .
$$

Если для некоторого $k$ выполняется равенство $R_{k}=R_{k+1}$, то $R_{k+1}=R_{k+i}$ для всех $i \geqslant 1$. Наименьшее $k$ с таким свойством называется глубиной орбитно-неустойчивого центра, а соответствующее $R_{k}$ - орбитно-неустойчивым чентром слоения $\mathscr{F}$. Обозначим глубину орбитно-неустойчивого центра через $D(\mathscr{F})$. Отметим, что мы не исключаем случая, когда $R_{k}=\varnothing$, т.е. слоение может иметь пустой орбитно-неустойчивый центр. Можно привести примеры слоений с любым $D(\mathscr{F}) \geqslant 0$ и либо пустым, либо непустым орбитно-неустойчивым центром.

Непосредственно из леммы 1 вытекает следующий результат (означающий, что глубина орбитно-неустойчивого центра является топологическим инвариантом).

Лемма 2. Пусть слоения $\mathscr{F}_{1}, \mathscr{F}_{2}$ топологически эквивалентны и имеют конечные глубины $D\left(\mathscr{F}_{1}\right), D\left(\mathscr{F}_{2}\right)$ орбитно-неустойчивых центров соответственно. Тогда имеем $D\left(\mathscr{F}_{1}\right)=D\left(\mathscr{F}_{2}\right)$.

Пусть $f(z)=\exp z$, и положим $f(f(\ldots(z)) \ldots)=f^{n}(z)$. Поскольку $f^{n}(z)$ является целой функцией, линии уровня ее мнимой части $\operatorname{Im} f^{n}(z)$ определяют параболическое слоение, которое мы обозначим через $\mathscr{F}_{n}$. Так как $f^{n}(z) \neq 0$, то слоение $\mathscr{F}_{n}$ не имеет особенностей. Покажем, что слоения $\mathscr{F}_{n}$ попарно топологически не эквивалентны. Для этого будет удобно продолжить $\mathscr{F}_{n}$ на сферу $S^{2}=\mathbb{R}^{2} \cup\{\infty\}$, считая присоединенную "бесконечную" точку особенностью $s_{0}$. Обозначим продолженное слоение на $S^{2}$ через $\widetilde{\mathscr{F}}_{n}$. Поскольку гомеоморфизм, осуществляющий топологическую эквивалентность слоений, переводит особенность в особенность, эквивалентность слоений $\mathscr{F}_{n}$ равносильна эквивалентности слоений $\widetilde{\mathscr{F}}_{n}$. 
Множество $\operatorname{Im} f^{n}(z)=c$ обозначим через $\mathscr{L}_{c}^{n}$. Оно состоит из не более чем счетного семейства слоев слоения $\mathscr{F}_{n}$, которые будут записываться в виде $L_{c, i}^{n}$.

Поскольку

$$
\operatorname{Im} f(z)=\operatorname{Im} \exp z=\exp x \sin y=c,
$$

слоение $\mathscr{F}_{1}$ состоит из слоений Рэба в бесконечных полосах с граничными слоями $L_{0, k}^{1}=$ $\{y=\pi k\}, k \in \mathbb{Z}$, см. рис.

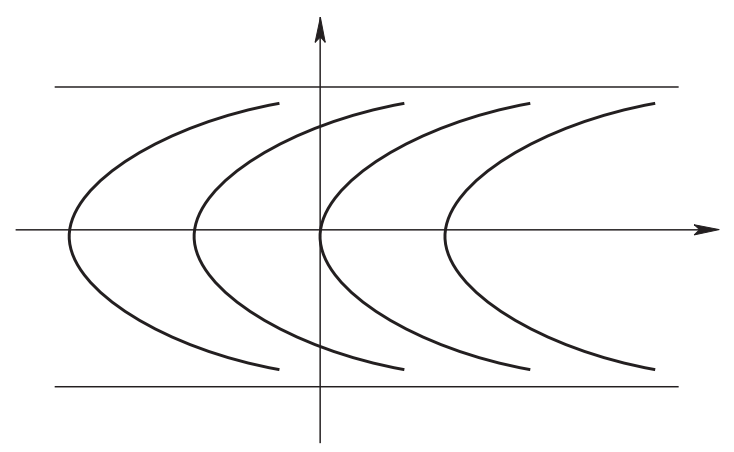

Рис.

Непосредственно проверяется, что $D\left(\widetilde{\mathscr{F}_{1}}\right)=1$.

Покажем, что слоение $\mathscr{F}_{n+1}$ определяется равенствами

$$
f\left(\operatorname{Re} f^{n}(z)\right) \sin \left(\operatorname{Im} f^{n}(z)\right)=c,
$$

где $c$ пробегает множество действительных чисел. Действительно,

$$
\begin{aligned}
\operatorname{Im} f^{n+1}(z) & =\operatorname{Im} \exp \left(f^{n}(z)\right)=\operatorname{Im} \exp \left(\operatorname{Re} f^{n}(z)+i \operatorname{Im} f^{n}(z)\right) \\
& =\exp \left(\operatorname{Re} f^{n}(z)\right) \sin \left(\operatorname{Im} f^{n}(z)\right)=f\left(\operatorname{Re} f^{n}(z)\right) \sin \left(\operatorname{Im} f^{n}(z)\right) .
\end{aligned}
$$

Из (1) следует, что

$$
\mathscr{L}_{\pi k}^{n} \subset \mathscr{L}_{0}^{n+1} \quad \forall n \in \mathbb{N}, k \in \mathbb{Z}
$$

В частности,

$$
\mathscr{L}_{\pi k}^{1} \subset \mathscr{L}_{0}^{2} \subset \cdots \subset \mathscr{L}_{0}^{n} \cdots .
$$

Сосредоточим наше внимание на полосе $S_{1}$, ограниченной прямыми $y=2 \pi$ и $y=3 \pi$, которые в силу (2) являются слоями всех слоений $\mathscr{F}_{n}$. Поскольку ограничение $\left.f\right|_{\operatorname{int}} S_{1}$ отображения $f$ на внутренность $\operatorname{int} S_{1}$ полосы $S_{1}$ есть диффеоморфизм на верхнюю полуплоскость, пересечение $S_{1} \cap f^{-1}\left(S_{1}\right) \stackrel{\text { def }}{=} S_{2}$ является областью в $S_{1}$, гомеоморфной $S_{1}$.

Из (1) получаем, что слоение $\mathscr{F}_{2}$ определяется равенствами $f\left(\operatorname{Re} f^{2}(z)\right) \sin \left(\operatorname{Im} f^{2}(z)\right)=c$. Так как отображение $f$ имеет вид $x^{\prime}=\operatorname{Re} f(z), y^{\prime}=\operatorname{Im} f(z)$, то $\left.f\right|_{S_{2}}$ переводит слоение $\mathscr{F}_{2}$ на полосе $S_{2}$ в слоение $\mathscr{F}_{1}$ на полосе $S_{1}$. Таким образом, $\mathscr{F}_{2}$ на $S_{2}$ является слоением Рэба в бесконечной полосе. Следовательно, ограничивающие $S_{2}$ слои $L_{2 \pi, 0}^{1}, L_{3 \pi, 0}^{1}$ слоения $\mathscr{F}_{2}$ орбитно-неустойчивы. С другой стороны, слои $L_{2 \pi, 0}^{1}, L_{3 \pi, 0}^{1}$ принадлежат множествам $\mathscr{L}_{2 \pi}^{1}, \mathscr{L}_{3 \pi}^{1}$ соответственно. Поэтому $L_{2 \pi, 0}^{1}, L_{3 \pi, 0}^{1}$ лежат внутри полосы $S_{1}$ и, следовательно, являются орбитно-устойчивыми слоями слоения $\mathscr{F}_{1}$. Отсюда получаем, что $D\left(\widetilde{\mathscr{F}}_{2}\right) \geqslant$ $D\left(\widetilde{\mathscr{F}_{1}}\right)+1$. Полностью аналогично показывается, что

$$
D\left(\widetilde{\mathscr{F}}_{n+1}\right) \geqslant D\left(\widetilde{\mathscr{F}}_{n}\right)+1
$$

(на самом деле можно показать, что имеет место равенство, но нам достаточно неравенства). Из леммы 2 вытекает, что слоения $\mathscr{F}_{n}$ попарно топологически не эквивалентны.

Авторы благодарят Д. В. Аносова за полезные обсуждения. 


\section{СПИСОК ЦИТИРОВАННОЙ ЛИТЕРАТУРЫ}

[1] W. Kaplan, Trans. Amer. Math. Soc., 63 (1948), 514-522. [2] A. А. Андронов, Е. А. Леонтович, И. И. Гордон, А. Г. Майер, Качественная теория динамических систем второго порядка, Наука, М., 1966.

Е. В. Жужома

Нижегородский государственный педагогический университет

E-mail: zhuzhoma@mail.ru

В. С. Медведев

НИИ прикладной математики и кибернетики,

Нижегородский государственный

университет им. Н. И. Лобачевского
Поступило

21.01.2008 